\title{
ADUBAÇÃO MINERAL, ORGÂNICA E ORGANOMINERAL: EFEITOS NA NUTRIÇÃO, PRODUTIVIDADE, PÓS-COLHEITA DA CEBOLA E NA FERTILIDADE DO SOLO
}

\author{
Mineral, organic and organomineral fertilization: effects on nutrition, yield, postharvest of onion and soil fertility
}

Fábio Satoshi Higashikawa ${ }^{1 *}$; Francisco Olmar Gervini de Menezes Júnior ${ }^{1}$

\author{
${ }^{1}$ Pesquisador; Estação Experimental de Ituporanga; Empresa de Pesquisa Agropecuária e Extensão Rural de Santa Catarina - \\ Epagri/Ituporanga - SC, Brasil. \\ fabiohigashikawa@epagri.sc.gov.br; franciscomenezes@epagri.sc.gov.br \\ ${ }^{*}$ Autor para correspondência
}

Artigo enviado em 15/03/2017, aceito em 12/04/2017 e publicado em 07/07/2017.

\begin{abstract}
RESUMO - A adubação orgânica, pelo efeito residual no solo, tem maior influência nos atributos de fertilidade do solo em relação a adubação mineral. O objetivo do presente estudo foi avaliar o efeito das adubações mineral, orgânica e organomineral na nutrição, produtividade, pós-colheita da cebola e na fertilidade do solo após o ciclo da cebola. Os tratamentos consistiram de diferentes doses e fontes de $\mathrm{N}: 0 \mathrm{~N}$ = tratamento sem aplicação de $\mathrm{N} ; 75 \mathrm{~N}-\mathrm{M}$ $=75 \mathrm{~kg} \mathrm{ha}{ }^{-1}$ de $\mathrm{N}$ na forma de nitrato de amônio (NA); $75 \mathrm{~N}-\mathrm{O}=75 \mathrm{~kg} \mathrm{ha}^{-1}$ de $\mathrm{N}$ na forma de EA; $75 \mathrm{~N}-\mathrm{OM}=75$ $\mathrm{kg} \mathrm{ha}^{-1}$ de $\mathrm{N}$ metade como NA e outra metade como esterco de aves (EA); $125 \mathrm{~N}-\mathrm{M}=125 \mathrm{~kg} \mathrm{ha}^{-1}$ de $\mathrm{N}$ na forma de $\mathrm{NA} ; 125 \mathrm{~N}-\mathrm{O}=125 \mathrm{~kg} \mathrm{ha}^{-1}$ de $\mathrm{N}$ na forma de EA; $125 \mathrm{~N}-\mathrm{OM}=125 \mathrm{~kg} \mathrm{ha}^{-1}$ de $\mathrm{N}$ metade como NA e outra metade como EA. Os diferentes tipos de adubações influenciaram na nutrição, mas não apresentaram diferença estatística na produtividade. Independentemente da fonte, os bulbos dos tratamentos que receberam maiores doses de $\mathrm{N}$ foram aqueles que apresentaram maiores perdas pós-colheita. De modo geral, a adubação orgânica (75N-O e 125N-O) proporcionou melhoria nos atributos físico-químicos do solo em relação a adubação convencional (75 N-M e $125 \mathrm{~N}$ $\mathrm{M})$.
\end{abstract}

Palavras-Chave - esterco de aves, resíduo orgânico, manejo de adubação, Allium cepa L., quimiometria

\begin{abstract}
Organic fertilization, due to the residual effect on the soil, has a greater influence on soil fertility attributes in relation to mineral fertilization. The objective of the present study was to evaluate the effect of mineral, organic and organomineral fertilization on nutrition, yield, onion post-harvest and on soil fertility after onion cultivation. The treatments consisted of different doses and sources of $\mathrm{N}: 0 \mathrm{~N}=$ treatment without application of $\mathrm{N}$; $75 \mathrm{~N}-\mathrm{M}=75 \mathrm{~kg} \mathrm{ha}^{-1}$ of $\mathrm{N}$ as ammonium nitrate $(\mathrm{NA}) ; 75 \mathrm{~N}-\mathrm{O}=75 \mathrm{~kg} \mathrm{ha}^{-1}$ of $\mathrm{N}$ as EA (poultry manure); $75 \mathrm{~N}-\mathrm{OM}=$ $75 \mathrm{~kg} \mathrm{ha}^{-1}$ of $\mathrm{N}$ half as NA and half as EA; $125 \mathrm{~N}-\mathrm{M}=125 \mathrm{~kg} \mathrm{ha}^{-1}$ of $\mathrm{N}$ as NA; $125 \mathrm{~N}-\mathrm{O}=125 \mathrm{~kg} \mathrm{ha}^{-1}$ of N as EA; $125 \mathrm{~N}-\mathrm{OM}=125 \mathrm{~kg} \mathrm{ha}^{-1}$ of $\mathrm{N}$ half as NA and other half as EA. The different types of fertilization influenced the nutrition, but did not present statistical difference in the yield. Regardless of the source, the bulbs of the treatments that received the highest doses of $\mathrm{N}$ were those that presented the highest post-harvest losses. In general, organic fertilization $(75 \mathrm{~N}-\mathrm{O}$ and $125 \mathrm{~N}-\mathrm{O})$ provided an improvement in soil physicochemical attributes in relation to conventional fertilization (75 N-M and $125 \mathrm{~N}-\mathrm{M})$.
\end{abstract}

Keywords - poultry manure, organic waste, fertilization management, Allium cepa L., chemometrics 


\section{INTRODUÇÃO}

A cebola (Allium repa L.) é uma das espécies oleráceas mais apreciadas e consumidas em todo o mundo. No Brasil a cebola é a terceira hortaliça mais importante em termos econômicos (EPAGRI, 2013).

$\mathrm{Na}$ literatura, são poucos os estudos que abordam o uso da adubação orgânica na produção de cebola (VIDIGAL et al., 2010; MENEZES JÚNIOR; GONÇALVES; KURTZ, 2013; MENEZES JÚNIOR; GONÇALVES; VIEIRA NETO, 2014). Os motivos por essa escassez de estudos pode ser o desequilíbrio e a variabilidade de nutrientes contidos nos resíduos orgânicos (WESTERMAN; BICUDO, 2005) e a restrição a utilização de materiais disponíveis local ou regionalmente pela limitação econômica do transporte de grandes quantidades de resíduos (HIGASHIKAWA; KURTZ, 2016). Com relação à adubação orgânica para produção de cebola também são escassos os trabalhos que avaliam o efeito residual desse tipo de adubação na fertilidade do solo (MENEZES JÚNIOR; GONÇALVES; KURTZ, 2013; SOUZA et al., 2013; LEE et al., 2014) e na conservação pós-colheita da cebola (SOUZA et al., 2013).

O cálculo da adubação orgânica é feito com base nas concentrações de N, P ou K (CQFS-RS/SC, 2016), pois é impossível considerar todos os nutrientes em conjunto para definir a dose de adubo orgânico. Devido a isso, opta-se por um dos nutrientes para se definir a dose (SILVA, 2008). Por causa do efeito residual da adubação orgânica é importante considerar a influência desta prática na fertilidade do solo. Pois, os atributos como o $\mathrm{pH}$, a saturação por bases e a capacidade de troca catiônica são influenciados pela adubação orgânica (BARBOSA et al., 2007; MENEZES JÚNIOR; GONÇALVES; KURTZ, 2013).

O objetivo do presente estudo foi avaliar os efeitos das adubações mineral, orgânica e organomineral na produtividade, nutrição e conservação pós-colheita da cebola e na fertilidade do solo após o ciclo da cebola.

\section{MATERIAL E MÉTODOS}

\section{Descrição da área experimental}

O trabalho foi conduzido na Epagri, Estação Experimental de Ituporanga, SC, nos meses de julho a novembro de 2015, utilizando-se mudas do cultivar Empasc 352 - Bola Precoce. A Estação Experimental de Ituporanga está situada a $27^{\circ} 25^{\prime} \mathrm{S}, 49^{\circ} 30^{\prime} \mathrm{W}$ com altitude de $475 \mathrm{~m}$ e clima subtropical úmido com verão quente - Cfa segundo Köppen (KOTTEK et al., 2006). O solo, Cambissolo Háplico (EMBRAPA, 2013) com textura argilosa (42 \% de argila), apresentou as seguintes características químicas: $\mathrm{pH}=$ 5,$1 ; \mathrm{H}+\mathrm{Al}=9,2 \mathrm{cmol}_{\mathrm{c}} \mathrm{dm}^{-3}$; capacidade de troca catiônica (CTC) a pH $7(\mathrm{~T})=22 \mathrm{cmol}_{\mathrm{c}} \mathrm{dm}^{-3} ; \mathrm{Ca}=8,8$ $\mathrm{cmol}_{\mathrm{c}} \mathrm{dm}^{-3} ; \mathrm{Mg}=3,7 \mathrm{cmol}_{\mathrm{c}} \mathrm{dm}^{-3} ; \mathrm{Al}=0,5 \mathrm{cmol}_{\mathrm{c}} \mathrm{dm}^{-3}$; $\mathrm{P}$ (Mehlich) $=39,6 \mathrm{mg} \mathrm{dm}{ }^{-3} ; \mathrm{K}=0,39 \mathrm{cmol}_{\mathrm{c}} \mathrm{dm}^{-3}$; Saturação por bases $(\mathrm{V} \%)=58,2 \%$; Soma de Bases = 12,83; matéria orgânica $(\mathrm{MO})=2,8 \%$. Durante o período do experimento foram observados altos volumes de precipitação devido ao fenômeno " $E l$ Niño" (Figura 1). Os dados de temperatura e precipitação foram obtidos da estação meteorológica da Epagri, Estação Experimental de Ituporanga, SC. Com exceção do mês de agosto, nos demais meses a precipitação foi acima da média histórica (EPAGRI, 2016a,b). Nos meses de setembro e outubro os volumes ultrapassaram mais de $100 \mathrm{~mm}$ acima da média histórica (EPAGRI, 2016b).

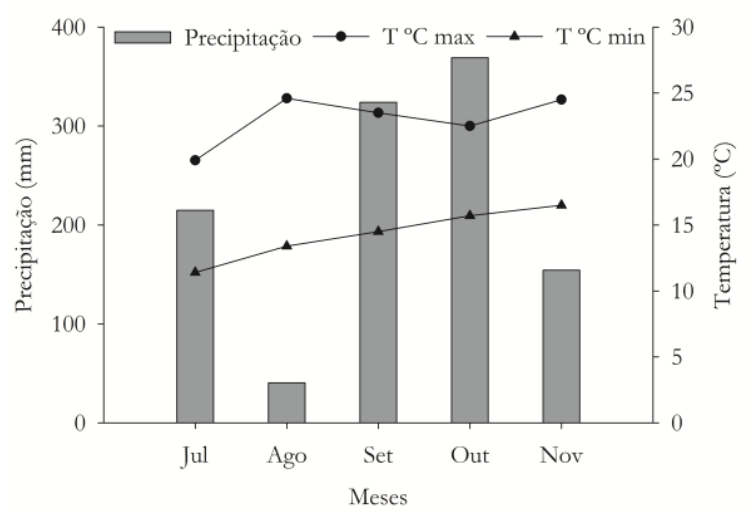

Figura 1. Precipitação mensal e médias das temperaturas máximas e mínimas referentes aos meses de julho a novembro de 2015. Epagri, Ituporanga, SC.

\section{Delineamento experimental, tratamentos e} caracterização do esterco de aves

O transplante das mudas, em julho de 2015, foi feito sob palha de adubação verde de verão consorciada de milheto (Pennisetum glaucum) e feijão miúdo (Vigna unguiculata (L.) Walp.), semeados respectivamente na densidade de 30 e $40 \mathrm{~kg} \mathrm{ha}^{-1}$. Em cada parcela de $9,6 \mathrm{~m}^{2}$ foi utilizado espaçamento de 35 $\mathrm{cm}$ entre linhas e $7 \mathrm{~cm}$ entre plantas, o equivalente a uma população de 400 mil plantas ha-1. O delineamento experimental foi $\mathrm{em}$ blocos ao acaso com quatro repetições e sete tratamentos. Os 
tratamentos foram respectivamente: $0 \mathrm{~N}=$ tratamento sem aplicação de $\mathrm{N} ; 75 \mathrm{~N}-\mathrm{M}=75 \mathrm{~kg} \mathrm{ha}^{-1}$ de $\mathrm{N}$ na forma de nitrato de amônio (NA); $75 \mathrm{~N}-\mathrm{O}=75 \mathrm{~kg} \mathrm{ha}^{-1}$ de $\mathrm{N}$ na forma de esterco de aves (EA); $75 \mathrm{~N}-\mathrm{OM}=$ $75 \mathrm{~kg} \mathrm{ha}^{-1}$ de $\mathrm{N}$ sendo metade como NA e outra metade como EA; $125 \mathrm{~N}-\mathrm{M}=125 \mathrm{~kg} \mathrm{ha}^{-1}$ de $\mathrm{N}$ na forma de NA; $125 \mathrm{~N}-\mathrm{O}=125 \mathrm{~kg} \mathrm{ha}^{-1}$ de $\mathrm{N}$ na forma $\mathrm{EA} ; 125 \mathrm{~N}-\mathrm{OM}=125 \mathrm{~kg} \mathrm{ha}^{-1}$ de $\mathrm{N}$ sendo metade na como NA e outra metade como EA. Sendo $\mathrm{M}=$ adubação mineral, $\mathrm{O}=$ adubação orgânica e $\mathrm{OM}=$ adubação organomineral. Os tratamentos diferiram pelas doses, fontes de $\mathrm{N}$ e pelos parcelamentos realizados. Os parcelamentos foram realizados de duas formas, em duas vezes (quando foi utilizada a dose de $75 \mathrm{~kg} \mathrm{ha}^{-1}$ de N, aplicada $50 \%$ no plantio e $50 \%$ aos 45 dias após transplante - DAT) e em quatro vezes (com uso de $125 \mathrm{~kg} \mathrm{ha}^{-1}$ de $\mathrm{N}$, com $15 \%$ da dose aplicado no plantio, $35 \%$ aos 30 e 60 DAT e $15 \%$ aos 90 DAT). As maiores doses foram aplicadas aos 30 e 60 DAT, pois são períodos que englobam a fase de rápido crescimento da planta e o início da bulbificação (KURTZ et al., 2016). As fontes de $\mathrm{N}$ utilizadas foram o nitrato de amônio (NA), o esterco de aves granulado (EA) e a mistura dessas duas fontes $(50 \%$ da dose de $\mathrm{N}$ com NA e $50 \%$ com EA). As adubações de cobertura, independente da fonte (orgânica ou mineral) foram feitas em superfície. O parcelamento teve por objetivo reduzir as perdas por escoamento superficial e de $\mathrm{N}$ por volatilização de amônia. Após as adubações de cobertura foram realizadas irrigações leves para acelerar a mineralização do EA e a disponibilidade de nutrientes para as plantas de cebola. Para atender as doses de fertilizante mineral foi utilizado NA com teor de nitrogênio de $27 \%$. O EA foi previamente caracterizado no Laboratório de Solos da Epagri, Estação Experimental de Ituporanga, SC, baseadas nas metodologias da Embrapa (2009). Os seguintes atributos foram determinados: $\mathrm{pH}$, condutividade elétrica (CE) e teores de $\mathrm{N}, \mathrm{P}, \mathrm{K}, \mathrm{Ca}$, $\mathrm{Mg}, \mathrm{Fe}, \mathrm{Zn}$ e B. Brevemente: determinação de $\mathrm{pH}$ e CE em extratos 1:5 (m/v), determinação de $\mathrm{N}$ pelo método de Kjeldab); digestão ácida de EA (ácido nítrico e ácido clorídrico) em bloco digestor e determinação de $\mathrm{P}$ e B por colorimetria, $\mathrm{K}$ por fotometria de chama e os demais nutrientes por espectrofotometria por absorção atômica. As características físico-químicas de EA foram: $\mathrm{pH}(7,5)$; CE $\left(10,47 \mathrm{mS} \mathrm{cm}^{-1}\right) ; \mathrm{N}(13,3 \mathrm{~g}$ $\left.\mathrm{kg}^{-1}\right)$; P $\left(8,3 \mathrm{~g} \mathrm{~kg}^{-1}\right)$; $\left(30,4 \mathrm{~g} \mathrm{~kg}^{-1}\right)$; Ca $\left(1,5 \mathrm{~g} \mathrm{~kg}^{-1}\right) ; \mathrm{Mg}$ $\left(0,7 \mathrm{~g} \mathrm{~kg}^{-1}\right) ; \mathrm{Fe}\left(3029 \mathrm{mg} \mathrm{kg}^{-1}\right) ; \mathrm{Zn}\left(27 \mathrm{mg} \mathrm{kg}^{-1}\right)$ e B (1,9 $\left.\mathrm{mg} \mathrm{kg}{ }^{-1}\right)$. As doses aplicadas de EA foram calculadas com base na fórmula proposta por CQFS-RS/SC (2016). As quantidades de EA para atender as doses de 75 e $125 \mathrm{~kg}$ de $\mathrm{N} \mathrm{ha}^{-1}$ foram respectivamente de 13,5 e
22,5 $\mathrm{t} \mathrm{ha}^{-1}$. Nos tratamentos que receberam somente EA $(75 \mathrm{~N}-\mathrm{O}$ e $125 \mathrm{~N}-\mathrm{O})$ não foi adicionada nenhuma fonte adicional de fertilizantes minerais. Nestes tratamentos o EA foi fonte única de nutrientes. Para os tratamentos que receberam somente NA como fonte de nitrogênio ( $75 \mathrm{~N}-\mathrm{M}$ e $125 \mathrm{~N}-\mathrm{M})$ foram adicionados o superfostato simples e o cloreto de potássio respectivamente nas doses de 80 e $90 \mathrm{~kg} \mathrm{ha}^{-1}$ de $\mathrm{P}_{2} \mathrm{O}_{5}$ e $\mathrm{K}_{2} \mathrm{O}$. Nos tratamentos que receberam adubação organomineral (75N-OM e $125 \mathrm{~N}-\mathrm{OM})$ não foi necessário complementar a adubação potássica, mas foi aplicado superfosfato simples para complementar o fornecimento de $80 \mathrm{~kg} \mathrm{ha}^{-1}$ de $\mathrm{P}_{2} \mathrm{O}_{5}$.

Coletas de amostras de folhas e de solos para análises da nutrição da cebola e da fertilidade do solo

$\mathrm{Na}$ primeira quinzena de outubro, no início da bulbificação, foram coletadas folhas jovens totalmente desenvolvidas de dez plantas por parcela. Imediatamente após a coleta das folhas, as amostras foram secas em estufas de circulação forçada de ar a $65^{\circ} \mathrm{C}$ até atingirem peso constante. Posteriormente, as amostras foram moídas em moinho tipo Willey com peneira de malha de $1 \mathrm{~mm}$. Após serem moídas as amostras foram analisadas para determinação dos teores de N, P, K, Ca, Mg, B, Cu, Fe, Mn e Zn no laboratório de Nutrição Vegetal da Embrapa Clima Temperado de acordo com as metodologias descritas em Malavolta, Vitti e Oliveira (1989). Brevemente: foi utilizado o método da digestão sulfúrica para extrair $\mathrm{N}$, digestão ácida a quente para extrair $\mathrm{P}, \mathrm{K}, \mathrm{Ca}, \mathrm{Mg}$, $\mathrm{Cu}, \mathrm{Fe}, \mathrm{Mn}$ e $\mathrm{Zn}$ e decomposição por via seca para extrair B. As determinações para os nutrientes foram feitas pelos seguintes métodos: semi-micro-Kjeldabl para N, colorimetria para $\mathrm{P}$ e $\mathrm{B}$ e por espectofotometria de absorção atômica para $\mathrm{K}, \mathrm{Ca}$, $\mathrm{Mg}, \mathrm{Cu}, \mathrm{Fe}, \mathrm{Mn}$ e Zn.

Em cada parcela, após a colheita da cebola, foram coletados cinco pontos aleatórios de subamostras para compor uma amostra composta de solo para realizar análise de fertilidade. Os seguintes atributos foram considerados para avaliar o efeito dos tipos de adubações na fertilidade do solo: $\mathrm{pH}$, matéria orgânica (MO), $\mathrm{H}+\mathrm{Al}$ (acidez potencial), capacidade de troca catiônica a $\mathrm{pH} 7,0(\mathrm{~T})$, saturação por bases $(\mathrm{V} \%)$, soma de bases (SB), condutividade elétrica (CE), e teores de $\mathrm{Na}, \mathrm{P}, \mathrm{K}, \mathrm{Ca}, \mathrm{Mg}, \mathrm{S}, \mathrm{Zn}, \mathrm{Cu}, \mathrm{Mn}, \mathrm{B}$ e Fe. As análises das amostras de solos foram feitas na Epagri, Laboratório de Solos da Estação Experimental de Ituporanga, SC, de acordo com as metodologias da CQFS-RS/SC (2016). Brevemente: o pH foi 
determinado em água na proporção 1:1 (v/v); a MO determinada por colorimetria; $\mathrm{H}+\mathrm{Al}, \mathrm{T}, \mathrm{V} \%$ e $\mathrm{SB}$ por cálculos; $\mathrm{CE}$ foi determinado no extrato obtido na proporção de 1:5 (v/v); Na, P, K, Zn, Cu e Mn foram extraídos pelo método Mehlich-1; Ca e $\mathrm{Mg}$ foram extraídos por $\mathrm{KCl} 1 \mathrm{~mol} \mathrm{~L}^{-1}$; $\mathrm{S}$ foi extraído por fosfato de cálcio e determinado por turbidimetria; B foi extraído por água quente e determinado por colorimetria assim como o P; o Fe foi extraído com oxalato de amônio $0,2 \mathrm{~mol} \mathrm{~L}^{-1} \mathrm{e}$ assim como os teores de $\mathrm{Na}, \mathrm{K}, \mathrm{Zn}, \mathrm{Cu}, \mathrm{Mn}$ também foi determinado por espectrofotometria de absorção atômica.

\section{Avaliação de produtividade e de conservação pós-colheita da cebola}

No final de novembro de 2015 foi feita a colheita da cebola e em seguida foi feita a avaliação da produtividade comercial dos tratamentos. Para avaliação da produtividade comercial foram considerados a massa fresca dos bulbos com diâmetro transversal maior que $35 \mathrm{~mm}$ e sem defeitos (apodrecimento, brotado, mofado, deformado ou com doenças). A classificação seguiu as normas estabelecidas para a cultura (BRASIL, 1995). Após a classificação e pesagem, os bulbos foram armazenados em estaleiros dentro de um galpão por 111 dias. Depois desse período os bulbos foram avaliados com relação a conservação pós-colheita. A avaliação póscolheita consistiu na determinação da porcentagem de perda de fitomassa fresca dos bulbos.

\section{Análise estatística}

Para realizar análise de variância e o teste de média foi utilizado o software Sisvar versão 5.6 (FERREIRA, 2011). Os dados foram submetidos à análise de variância e as médias comparadas pelo teste de Scott-Knott $(\mathrm{p}<0,05)$. As análises de componentes principais (ACP) e de agrupamento hierárquico (AAH) foram feitas com o intuito de visualizar os padrões dos agrupamentos dos atributos físico-químicos em cada tipo de adubação. O conjunto de dados utilizados para as análises mencionadas foram as médias dos atributos físico-químicos analisados que foram previamente autoescaladas para realização das análises de ACP e AAH. O software utilizado para a realização dessas análises foi o Chemoface versão 1.61 (NUNES et al., 2012).

\section{RESULTADOS E DISCUSSÃO}

Efeitos dos tipos de adubações na nutrição de cebola
Com exceção dos teores de $\mathrm{N}$ e de $\mathrm{Fe}$, não houve diferença estatística entre os tratamentos para os teores dos demais nutrientes no tecido foliar (Tabela 1). Os teores de $\mathrm{Ca}, \mathrm{Mg}$, Fe e $\mathrm{Mn}$ foram abaixo da faixa adequada para todos os tratamentos. Os teores de $\mathrm{N}$ nos tratamentos $0 \mathrm{~N}$ e $75 \mathrm{~N}-\mathrm{M}$ também estavam abaixo dos valores considerados ideais (MENEZES JÚNIOR; KURTZ, 2016). Com relação ao teor de $\mathrm{N}$, os tratamentos que receberam somente esterco de aves na adubação ( $75 \mathrm{~N}-\mathrm{O}$ e $125 \mathrm{~N}-\mathrm{O})$ e aquele que recebeu a maior dose de nitrato de amônio $(125 \mathrm{~N}-\mathrm{M})$ apresentaram teores dentro da faixa ideal. Possivelmente, a presença de $\mathrm{N}$ na forma orgânica nos tratamentos $75 \mathrm{~N}-\mathrm{O}$ e $75-\mathrm{OM}$ possibilitou a liberação mais lenta de $\mathrm{N}$ para a cebola e menor perda por lixiviação em relação ao tratamento $75 \mathrm{~N}-\mathrm{M}$ que tem fonte exclusivamente mineral (nitrato de amônio). $\mathrm{O}$ excesso de chuvas (Figura 1) aliada a menor quantidade de $\mathrm{N}$ fornecida e a rápida liberação de $\mathrm{N}$ pelo fertilizante mineral, pode ter resultado em menor teor deste nutriente no tecido das plantas do tratamento $75 \mathrm{~N}-\mathrm{M}$. De modo que, com exceção de $0 \mathrm{~N}$ e $75 \mathrm{~N}-\mathrm{M}$, não houve diferença estatística dos teores de $\mathrm{N}$ para os demais tratamentos. A adubação orgânica possivelmente manteve os teores de nitrato no solo mais estáveis durante o ciclo da cultura. Enquanto que a adubação mineral pode apresentar oscilações e rápidas mudanças na concentração de $\mathrm{N}$ na solução do solo (LEE, 2010). Pelo fato dos teores de $\mathrm{N}$ no tecido foliar dos tratamentos com EA terem diferido estatisticamente da testemunha $(0 \mathrm{~N})$ e serem estatisticamente igual ao tratamento convencional $(125 \mathrm{~N}-\mathrm{M})$, pode-se inferir que o parcelamento do EA não prejudicou a nutrição da cebola com $\mathrm{N}$. Possivelmente, após a aplicação de esterco houve liberação e disponibilidade de nitrato e amônio para as plantas (EGHBALL et al., 2002). Pois, depois da adubação de cobertura foi feita em seguida a irrigação dos experimentos para acelerar a mineralização do EA e disponibilizar nutrientes para a cebola. Assim, com o aumento da umidade do solo houve um aumento na taxa de mineralização do esterco (EGHBALL et al., 2002). Além disso, a irrigação logo após a aplicação de adubos orgânicos é uma forma de reduzir as perdas de $\mathrm{N}$ por volatilização (CQFS-RS/SC, 2016). Com exceção dos tratamentos $0 \mathrm{~N}$ e $75 \mathrm{~N}-\mathrm{M}$ os demais teores apresentados por outros tratamentos (Tabela 1) ficaram dentro da faixa de 23 a $38 \mathrm{~g} \mathrm{~kg}^{-1}$ e de 23 a $34 \mathrm{~g}$ $\mathrm{kg}^{-1}$ observados para a cultivar Empasc 352 - Bola Precoce em sistema de plantio direto respectivamente por Kurtz et al. (2013) e Menezes Júnior e Kurtz (2016). 
Tabela 1. Teores de macro e micronutrientes de tecido foliar de cebola em função dos tipos de adubações

\begin{tabular}{|c|c|c|c|c|c|c|c|c|c|c|}
\hline Tratamento & $\mathrm{N}$ & $\mathrm{P}$ & K & $\mathrm{Ca}$ & $\mathrm{Mg}$ & B & $\mathrm{Cu}$ & $\mathrm{Fe}$ & $\mathrm{Mn}$ & $\mathrm{Zn}$ \\
\hline $0 \mathrm{~N}$ & $21,23 \mathrm{~b}$ & $3,98^{\mathrm{ns}}$ & $22,13^{\text {ns }}$ & $4,88^{\mathrm{ns}}$ & $1,33^{\mathrm{ns}}$ & $20,00^{\mathrm{ns}}$ & $6,00^{\mathrm{ns}}$ & $33,75 \mathrm{~b}$ & $23,25^{\mathrm{ns}}$ & $10,75^{\mathrm{ns}}$ \\
\hline $75 \mathrm{~N}-\mathrm{M}$ & $22,63 \mathrm{~b}$ & 4,10 & 22,03 & 3,50 & 1,30 & 20,50 & 6,25 & $34,25 \mathrm{~b}$ & 25,50 & 10,50 \\
\hline $75 \mathrm{~N}-\mathrm{O}$ & $26,40 \mathrm{a}$ & 4,38 & 23,13 & 4,40 & 1,43 & 24,25 & 7,50 & $28,25 \mathrm{~b}$ & 23,75 & 10,75 \\
\hline $75 \mathrm{~N}-\mathrm{OM}$ & $24,95 \mathrm{a}$ & 4,48 & 22,90 & 4,20 & 1,55 & 23,50 & 6,75 & $40,00 \mathrm{~b}$ & 23,75 & 9,75 \\
\hline $125 \mathrm{~N}-\mathrm{M}$ & $27,20 \mathrm{a}$ & 4,18 & 22,28 & 4,65 & 1,50 & 19,00 & 7,00 & $34,25 \mathrm{~b}$ & 24,75 & 10,25 \\
\hline $125 \mathrm{~N}-\mathrm{O}$ & $26,28 \mathrm{a}$ & 4,78 & 23,38 & 4,73 & 1,45 & 22,25 & 6,75 & $34,00 \mathrm{~b}$ & 24,25 & 11,00 \\
\hline $125 \mathrm{~N}-\mathrm{OM}$ & $24,83 \mathrm{a}$ & 4,18 & 21,80 & 3,98 & 1,30 & 22,50 & 7,00 & $51,67 \mathrm{a}$ & 24,00 & 11,00 \\
\hline Faixa adequada* & $23-34 * *$ & $2-4$ & $20-50$ & $7-30$ & $2-4$ & $10-50$ & $6-20$ & 60-300 & $30-200$ & $10-50$ \\
\hline $\begin{array}{l}\text { 0N = tratamento se } \\
75 \mathrm{~N}-\mathrm{OM}=75 \mathrm{~kg} \\
125 \mathrm{~N}-\mathrm{O}=125 \mathrm{~kg} \mathrm{~h} \\
\text { mesma letra minúsc } \\
\text { *Valores considerad } \\
\text { Grande do Sul e de }\end{array}$ & $\begin{array}{l}\text { aplicação } \\
\text { de N se }\end{array}$ & 2FS-1 & $75 \mathrm{~kg}$ & $\begin{array}{l}\mathrm{N} \mathrm{na} \\
\text { meta }\end{array}$ & a de & o de am & (NA); & $\begin{array}{l}\mathrm{J}-\mathrm{O}=75 \\
\mathrm{I}=125\end{array}$ & $\begin{array}{l}\mathrm{g} \mathrm{ha}^{-1} \text { de } \\
h^{-1} \text { de }\end{array}$ & a forma \\
\hline
\end{tabular}

Efeitos dos tipos de adubações na produtividade de cebola

Não houve diferença estatística entre as produtividades dos tratamentos estudados (Figura 2). Possivelmente, o excesso de chuvas (Figura 1) reduziu o efeito dos diferentes tipos de adubações dos tratamentos. Gonçalves, Sousa e Silva (2003) também verificaram produtividades de cebola semelhantes entre tratamentos com fornecimento de $\mathrm{N}$ por fonte mineral ou orgânica. De qualquer modo, mesmo com o excesso de chuvas, foi possível obter médias de produtividades maiores (Figura 2) que a média nacional de 25,5 $\mathrm{t} \mathrm{ha}^{-1} \mathrm{e}$ a média estadual de 16,9 t ha-1 (IBGE, 2016). Deve-se enfatizar que apesar do tratamento $0 \mathrm{~N}$ não ter recebido adubação nitrogenada sua produtividade não diferiu dos demais. O que se deve, provavelmente, a contribuição do nitrogênio presente na matéria orgânica do solo (GONÇALVES et al., 2009) somado ao fornecimento do nutriente pela adubação orgânica com o feijão miúdo. Com efeito, estudos indicam que o feijão miúdo tem a capacidade de fixar de 74 a $116 \mathrm{~kg}$ de $\mathrm{N}_{\text {ha }}{ }^{-1}$ (ARAÚJO; WATT, 1988; AWONAIKE et al., 1990). Portanto, dependendo da necessidade da cultura e condições edafoclimáticas, a adubação verde com a referida leguminosa poderá substituir em parte ou até totalmente a adubação nitrogenada, o que explicaria a homogeneização das produtividades entre os tratamentos. No entanto, o teor de $\mathrm{N}$ no tecido ficou abaixo da faixa considerada ideal, o que indica menor disponibilidade de nitrogênio pelas fontes orgânicas (matéria orgânica do solo e leguminosa) no início da bulbificação em comparação aos demais tratamentos. Contudo, insuficiente para reduzir a produtividade. As médias de produtividades dos tratamentos foram em tha-1: 26,5 (0N); $30,5(75 \mathrm{~N}-\mathrm{M})$; 31,0 (75N-O); 26,6 (75N-OM); 28,27 (125N-M); 30,5 $(125 \mathrm{~N}-\mathrm{O})$ e 30,4 (125N-OM). As produtividades alcançadas neste estudo estão dentro da faixa encontrada na literatura para o cultivar Empasc 352 Bola Precoce que variou de 22 a $43 \mathrm{t} \mathrm{ha}^{-1}$ em experimentos que receberam tratamento fitossanitário convencional (KURTZ; ERNANI, 2010; KURTZ et al., 2013; MENEZES JÚNIOR; GONÇALVES; VIEIRA NETO, 2014). 


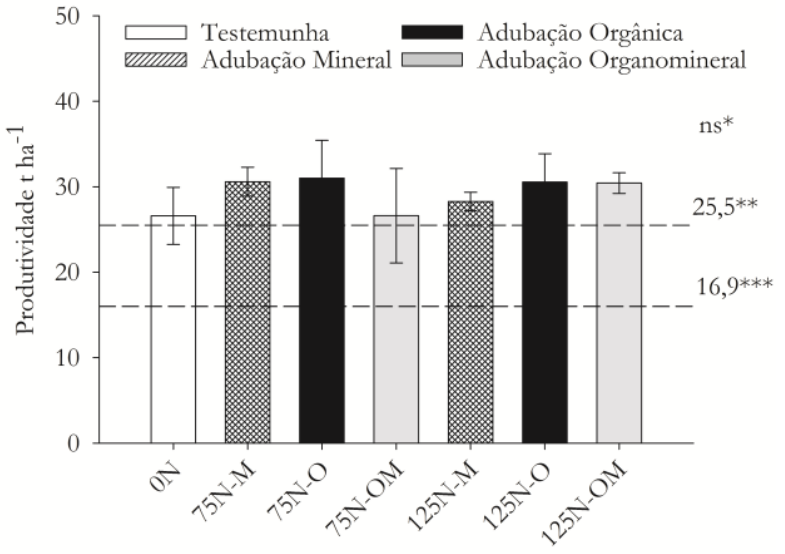

Tratamentos

Figura 2. Produtividade de cebola em função do tipo de adubação. $0 \mathrm{~N}=$ tratamento sem aplicação de $\mathrm{N} ; 75 \mathrm{~N}-\mathrm{M}$ $=75 \mathrm{~kg} \mathrm{ha}^{-1} \mathrm{de} \mathrm{N}$ na forma de nitrato de amônio (NA); $75 \mathrm{~N}-\mathrm{O}=75 \mathrm{~kg} \mathrm{ha}^{-1}$ de $\mathrm{N}$ na forma de EA; $75 \mathrm{~N}-\mathrm{OM}=75$ $\mathrm{kg} \mathrm{ha}^{-1}$ de $\mathrm{N}$ sendo metade como NA e outra metade como esterco de aves (EA); $125 \mathrm{~N}-\mathrm{M}=125 \mathrm{~kg} \mathrm{ha}^{-1}$ de $\mathrm{N}$ na forma de NA; $125 \mathrm{~N}-\mathrm{O}=125 \mathrm{~kg} \mathrm{ha}^{-1}$ de $\mathrm{N}$ na forma EA; $125 \mathrm{~N}-\mathrm{OM}=125 \mathrm{~kg} \mathrm{ha}^{-1}$ de $\mathrm{N}$ sendo metade como NA e outra metade como EA. ns* = não significativo pelo teste de $\mathrm{F}$ a $5 \%$ de probabilidade. $* *$ e $* * *=$ Se referem respectivamente a média de produtividade nacional e do estado de Santa Catarina do ano de 2015 (IBGE, 2016).

\section{Efeito dos tipos de adubações na cebola após} armazenamento

Após 111 dias de armazenamento os bulbos dos tratamentos que receberam as maiores quantidades de $\mathrm{N}$, independentemente da fonte, apresentaram as maiores perdas de massa fresca (Figura 3). Similarmente, Kurtz et al. (2013) também verificaram o efeito negativo do incremento das doses de $\mathrm{N}$ na conservação dos bulbos do cultivar Empasc 352 - Bola Precoce. As perdas pós-colheita foram elevadas devido ao excesso de chuvas durante o ciclo da cebola (Figura 1) que favoreceu o apodrecimento dos bulbos durante o armazenamento. Por essa razão, se o objetivo for armazenar os bulbos em anos com excesso de chuvas, é aconselhável reduzir o fornecimento de $\mathrm{N}$ para as plantas (KURTZ; ERNANI, 2012). As perdas póscolheita deste estudo, devido ao excesso de chuvas (Figura 1) variaram de 57 a $72 \%$ e foram bem mais elevadas do que as perdas de 16 a $41 \%$ apresentadas por Kurtz e Ernani (2010) após 145 dias de armazenamento dos bulbos.

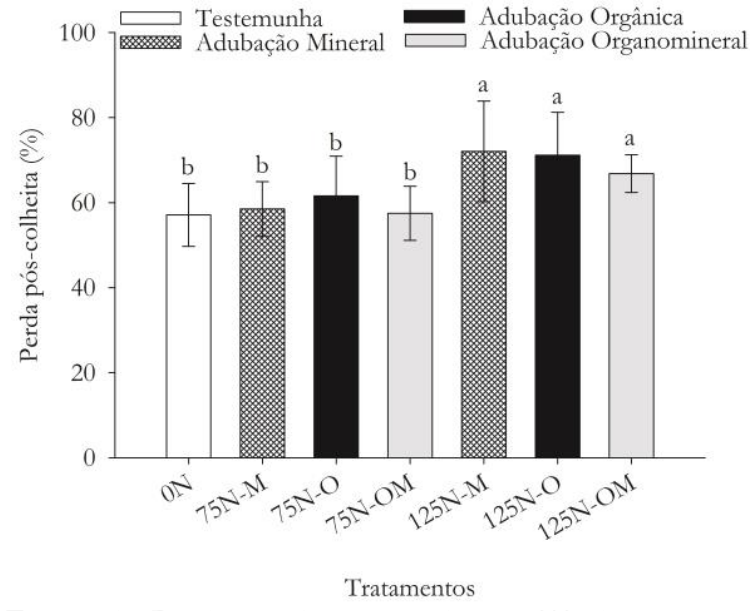

Figura 3. Perda de fitomassa fresca 111 dias após a colheita dos bulbos. $0 \mathrm{~N}=$ tratamento sem aplicação de $\mathrm{N} ; 75 \mathrm{~N}-\mathrm{M}=75 \mathrm{~kg} \mathrm{ha}^{-1}$ de $\mathrm{N}$ na forma de nitrato de amônio (NA); $75 \mathrm{~N}-\mathrm{O}=75 \mathrm{~kg} \mathrm{ha}^{-1}$ de $\mathrm{N}$ na forma de EA; $75 \mathrm{~N}-\mathrm{OM}=75 \mathrm{~kg} \mathrm{ha}^{-1}$ de $\mathrm{N}$ sendo metade como NA e outra metade como esterco de aves (EA); $125 \mathrm{~N}-\mathrm{M}=125$ $\mathrm{kg} \mathrm{ha}^{-1}$ de N na forma de NA; $125 \mathrm{~N}-\mathrm{O}=125 \mathrm{~kg} \mathrm{ha}^{-1} \mathrm{de}$ $\mathrm{N}$ na forma EA; $125 \mathrm{~N}-\mathrm{OM}=125 \mathrm{~kg} \mathrm{ha}^{-1}$ de $\mathrm{N}$ sendo metade como NA e outra metade como EA. Médias seguidas de mesma letra minúscula não diferem entre si pelo teste de Scott-Knott $(\mathrm{p}<0,05)$.

Efeito dos tipos de adubações na fertilidade do solo pós-colheita

Os tratamentos que receberam as maiores doses de fertilizante na forma orgânica $(75 \mathrm{~N}-\mathrm{O}, 125$ $\mathrm{OM}$ e $125 \mathrm{~N}-\mathrm{O})$ foram aqueles que apresentaram os maiores valores médios para a maioria dos atributos de fertilidade do solo avaliados após a colheita (Figura 4.) Portanto, foi observado que o incremento de dose do fertilizante orgânico favorece os atributos do solo. Consequententemente, a utilização de adubos orgânicos conforme a recomendação técnica foi benéfica para a fertilidade do solo (CQFS-RS/SC, 2016). Provavelmente, isso se deve ao maior efeito residual do esterco de aves (EA) no solo em relação aos tratamentos que receberam nitrato de amônio (NA). Para ser benéfico, o efeito residual no solo da aplicação do fertilizante orgânico deverá ser considerado no manejo de adubação da cultura subsequente a ser implantada na mesma área (BARBOSA et al., 2007). Caso contrário, podem ser provocados desequlíbrios nutricionais (SOUZA; ALCÂNTARA, 2008). Similarmente a este estudo, Menezes Júnior, Gonçalves e Kurtz (2013) também verificaram aumento nos valores de $\mathrm{MO}, \mathrm{pH}, \mathrm{P}, \mathrm{K}, \mathrm{Ca}$ e $\mathrm{V} \%$ em análises de 
amostras de solo coletadas após a colheita de cebola nos tratamentos que receberam a aplicação de esterco de peru, esterco bovino e biofertilizante a base de esterco bovino em relação ao tratamento convencional que recebeu fertilizantes minerais. A utilização de EA na adubação orgânica, além da melhoria da fertilidade do solo, permitiu a reciclagem de nutrientes e a redução do uso de fertilizantes minerais (HIGASHIKAWA; KURTZ, 2016).

Recomenda-se que em trabalhos futuros, em experimentos de longa duração, sejam avaliados o efeito da aplicação sucessiva de esterco de aves na fertilidade do solo.

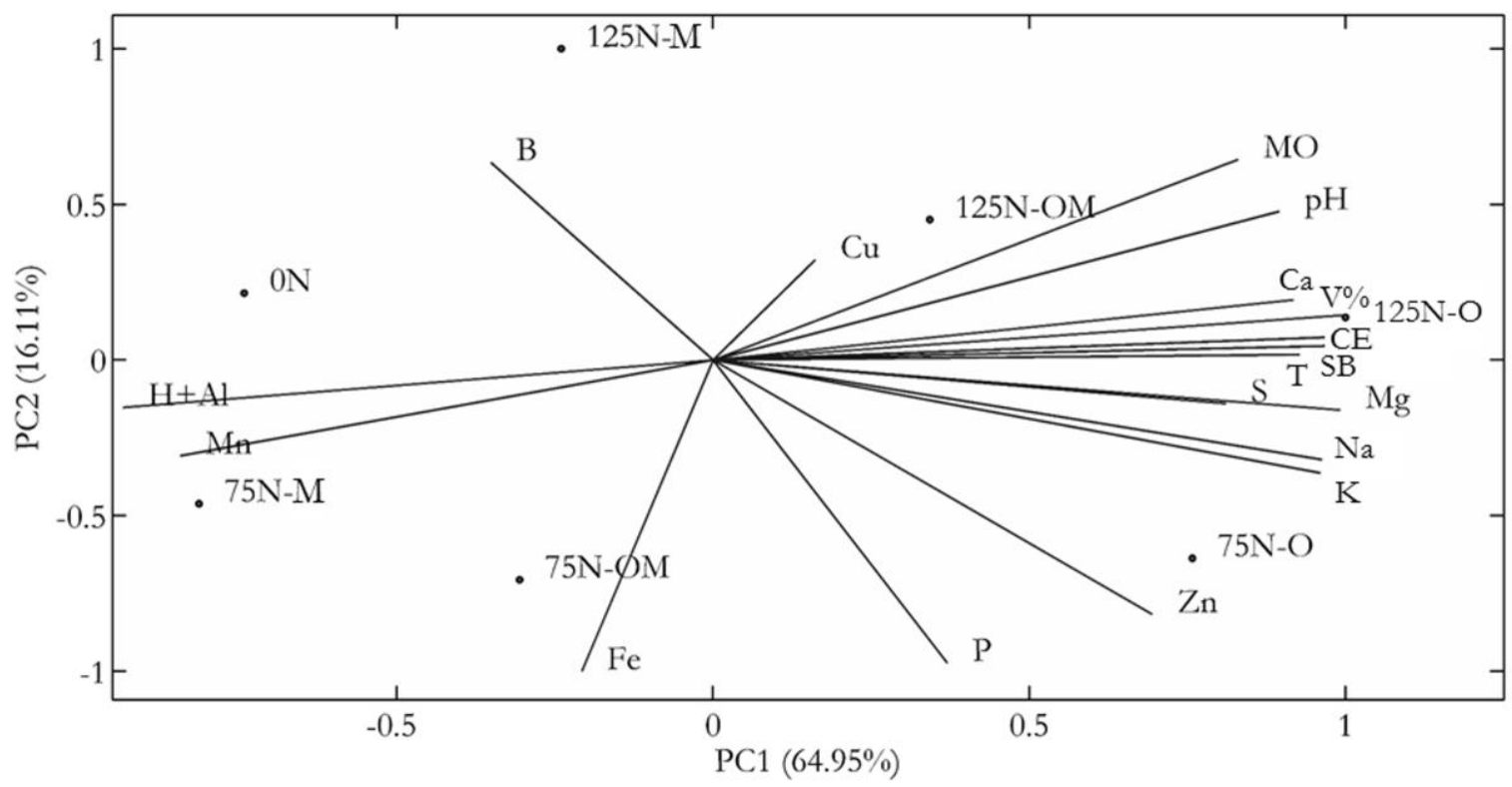

Figura 4. Análise de componentes principais de atributos físico-químicos de solo adubado com fontes mineral, orgânica e organomineral. PC1 = componente principal 1. PC2 = componente principal 2. As linhas representam os atributos físico-químicos e os pontos são referentes aos tratamentos. $0 \mathrm{~N}=$ tratamento sem aplicação de $\mathrm{N} ; 75 \mathrm{~N}-\mathrm{M}=75$ $\mathrm{kg} \mathrm{ha}^{-1}$ de $\mathrm{N}$ na forma de nitrato de amônio (NA); $75 \mathrm{~N}-\mathrm{O}=75 \mathrm{~kg} \mathrm{ha}^{-1}$ de $\mathrm{N}$ na forma de EA; $75 \mathrm{~N}-\mathrm{OM}=75 \mathrm{~kg} \mathrm{ha}{ }^{-1} \mathrm{de}$ $\mathrm{N}$, sendo metade como NA e outra metade como esterco de aves (EA); $125 \mathrm{~N}-\mathrm{M}=125 \mathrm{~kg} \mathrm{ha}^{-1}$ de N na forma de NA; $125 \mathrm{~N}-\mathrm{O}=125 \mathrm{~kg} \mathrm{ha}^{-1}$ de $\mathrm{N}$ na forma EA; $125 \mathrm{~N}-\mathrm{OM}=125 \mathrm{~kg} \mathrm{ha}^{-1}$ de $\mathrm{N}$ sendo metade como NA e outra metade como EA. $\mathrm{pH}=$ potencial hidrogeniônico; $\mathrm{MO}=$ matéria orgânica; $\mathrm{H}+\mathrm{Al}=$ acidez potencial; $\mathbf{T}=$ capacidade de troca catiônica a $\mathrm{pH} 7,0 ; \mathrm{V} \%$ = saturação por bases, $\mathrm{SB}=$ soma de bases; $\mathrm{CE}=$ condutividade elétrica; $\mathrm{Na}, \mathrm{P}, \mathrm{K}, \mathrm{Ca}, \mathrm{Mg}, \mathrm{S}$, $\mathrm{Zn}, \mathrm{Cu}, \mathrm{Mn}, \mathrm{B}$ e $\mathrm{Fe}=$ são teores dos respectivos elementos.

$\mathrm{Na}$ Figura 5 os tratamentos se diferenciaram em quatro grupos considerando a distância Euclidiana fixada em quatro. O primeiro grupo é formado por $125 \mathrm{~N}-\mathrm{O}$ e $75 \mathrm{~N}-\mathrm{O}$ que foram os tratamentos que receberam a maiores quantidades de $\mathrm{EA}$. O segundo grupo é formado por $125 \mathrm{~N}-\mathrm{OM}$ e $125 \mathrm{~N}-\mathrm{M}$ que apresentaram valores intermediários em relação aos valores médios dos atributos (Figura 4). O tratamento $0 \mathrm{~N}$ forma o terceiro grupo e se caracteriza por não ter recebido fertilização nitrogenada e por essa razão apresenta comportamento distinto dos demais grupos mencionados anteriormente, mas é semelhante ao quarto grupo. Este quarto grupo é formado por $75 \mathrm{~N}$ $\mathrm{OM}$ e $75 \mathrm{~N}-\mathrm{M}$ e se assemelham ao tratamento $0 \mathrm{~N}$ pelo fato de terem recebido respectivamente, as menores doses de $\mathrm{N}$ na forma organomineral e mineral. 


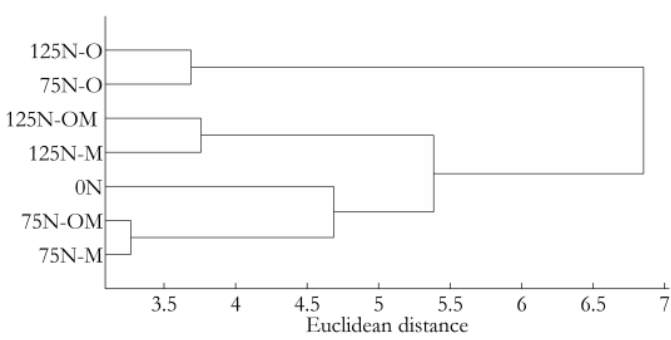

Figura 5. Dendrograma resultante da análise hierárquica de agrupamentos que representa a formação de grupos segundo os tipos de adubações realizadas. $0 \mathrm{~N}=$ tratamento sem aplicação de N; $75 \mathrm{~N}-\mathrm{M}=75 \mathrm{~kg} \mathrm{ha}^{-1} \mathrm{de}$ $\mathrm{N}$ na forma de nitrato de amônio (NA); $75 \mathrm{~N}-\mathrm{O}=75 \mathrm{~kg}$ $\mathrm{ha}^{-1}$ de $\mathrm{N}$ na forma de EA; $75 \mathrm{~N}-\mathrm{OM}=75 \mathrm{~kg} \mathrm{ha}^{-1}$ de N sendo metade como NA e outra metade como esterco de aves (EA); $125 \mathrm{~N}-\mathrm{M}=125 \mathrm{~kg} \mathrm{ha}^{-1}$ de $\mathrm{N}$ na forma de NA; $125 \mathrm{~N}-\mathrm{O}=125 \mathrm{~kg} \mathrm{ha}^{-1}$ de $\mathrm{N}$ na forma EA; $125 \mathrm{~N}-\mathrm{OM}=$ $125 \mathrm{~kg} \mathrm{ha}^{-1}$ de N sendo metade como NA e outra metade como EA.

\section{CONCLUSÕES}

Os teores de $\mathrm{N}$ no tecido foliar foram menores para os tratamentos $0 \mathrm{~N}$ e $75 \mathrm{~N}$.

Os teores foliares de $\mathrm{Ca}, \mathrm{Mg}, \mathrm{Fe}$ e $\mathrm{Mn}$ foram abaixo da faixa adequada para todos os tratamentos.

A produtividade de cebola foi similar entre os tratamentos.

As perdas pós-colheita dos bulbos foram incrementadas com as doses de $\mathrm{N}$.

A adubação orgânica proporcionou melhoria nos atributos físico-químicos do solo em relação a adubação mineral.

O efeito na fertilidade do solo, em relação a adubação organomineral, foi influenciada pela dose de esterco de aves.

\section{AGRADECIMENTOS}

A toda equipe de campo e do laboratório de Solos da Estação Experimental de Ituporanga pelas valiosas contribuições durante a condução do experimento e nas análises laboratoriais

$$
\text { À FAPESC projeto }
$$

FRANCISCOJUNIOR/FAPESC/TO 2013TR4003 pelo apoio financeiro.

\section{REFERÊNCIAS}

ARAÚJO, J.P.P.; WATT, E.E. Caupi no Brasil. Brasília: IITA/Embrapa, 1988. 722p.
AWONAIKE, K.O.; KUMARASINGHE, K.S.; DANSO, S.K.A. Nitrogen fixation and yield of cowpea (Vigna unguiculata) as influenced by cultivar and Bradyrhizobium strain. Fiels Crops Research, v.24, n. 1-3 p. 163-171, 1990.

BARBOSA, G.M.C.; TAVARES FILHO, J.; BRITO, O.R.; FONSECA, I.C.B. Efeito residual do lodo de esgoto na produtividade do milho safrinha. Revista Brasileira de Ciência do Solo, v. 31, n. 3, p. 601-605, jun. $2007 . \quad$ Disponível em: $<$ http://www.scielo.br/scielo.php?script=sci_arttext\& pid $=\mathrm{S} 0100$ -

$06832007000300020 \& \operatorname{lng}=\mathrm{pt} \& \mathrm{nrm}=\mathrm{i}$ so\&tlng $=\mathrm{pt}>$. Acesso em: 27 jun. 2016.

BRASIL. 1995. Ministério da Agricultura, Abastecimento e Reforma Agrária. Portaria n. 529 de 18 de ago. 1995. Diário Oficial da República Federativa do Brasil. Brasília, 1 de set. 1995, Seção 1:13513.

CQFS-RS/SC. Manual de calagem e adubação para os estados do rio grande do sul e de santa catarina. 11. ed. Santa Maria: Sociedade Brasileira de Ciência do Solo - Núcleo Regional Sul, 2016.

EGHBALL, B.; WIENHOLD, B. J.; GILLEY, J. E.; EIGENBERG, R. A. Mineralization of manure nutrients. Journal of Soil and Water Conservation, v. 57, n. 6, p. 470-473, 1 nov. 2002. Disponível em: <http://www.jswconline.org/content/57/6/470.short >. Acesso em: 21 jan. 2015.

EMBRAPA. Manual de análises químicas de solos, plantas e fertilizantes. 2. ed. Brasília: Embrapa Informação Tecnológica, 2009.

EMBRAPA. Sistema brasileiro de classificação de solos. 3. ed. Brasília: Embrapa Informação Tecnológica, 2013.

EPAGRI. Sistema de produção para a cebola: santa catarina. 4. ed. Florianópolis: Gerência de Marketing e Comunicação (GMC)/Epagri, 2013.

EPAGRI. Boletim ambiental. sintese trimestral: inverno 2015. Florianópolis: Epagri. Documentos, 253, 2016 a.

EPAGRI. Boletim ambiental. sintese trimestral: primavera 2015. Florianópolis: Epagri. Documentos, 257, 2016b. 
FERREIRA, D.F. Sisvar: a computer statistical analysis system. Ciência e Agrotecnologia, v. 35, n. 6, p. 1039-1042, 2011. Disponível em: <http:/ /www.scielo.br/scielo.php?script=sci_arttext\& pid=S1413-

$70542011000600001 \& \operatorname{lng}=\mathrm{en} \& \mathrm{nrm}=\mathrm{iso} \& \operatorname{lng}=\mathrm{en}>$.

Acesso em: 25 jan. 2013.

GONÇALVES, P. A. de S.; SILVA, C. R. S. e. Impacto da adubação orgânica sobre a incidência de tripes em cebola. Horticultura Brasileira, v. 21, n. 3, p. 459-463, set. $2003 . \quad$ Disponível em: $<$ http:/ /www.scielo.br/scielo.php?script=sci_arttext\& pid $=$ S0102-

05362003000300009\&lng $=$ en\&nrm $=$ iso\&tlng $=\mathrm{pt}>$.

Acesso em: 31 mar. 2017.

GONÇALVES, P. A. de S.; WORDELL FILHO, J. A.; KURTZ, C. Efeitos da adubação sobre a incidência de tripes e míldio e na produtividade da cultura da cebola. Agropecuária Catarinense, v. 22, n. 1, p. 57-60, 2009.

HIGASHIKAWA, F. S.; KURTZ, C. Manejo do solo, correção e adubação. In: MENEZES JÚNIOR, F. O. G.; MARCUZZO, L. L. (Ed.). Manual de prática agrícolas: guia para a sustentabilidade das lavouras de cebola do estado de Santa Catarina. 1. ed. Florianópolis: Departamento Estadual de Marketing e Comunicação (DEMC) / Epagri, 2016. p. 49-60.

IBGE. Indicadores IBGE: estatística da produção agrícoloa dezembro 2016. Rio de Janeiro - RJ: IBGE, 2016.

KOTTEK, M.; GRIESER, J.; BECK, C.; RUDOLF, B.; RUBEL, F. World Map of the Köppen-Geiger climate classification updated. Meteorologische Zeitschrift, v. 15, n. 3, p. 259-263, 1 jun. 2006. Disponível em: $<$ http:/ /openurl.ingenta.com/content/ xref?genre $=$ arti cle\&issn $=0941$ -

$2948 \&$ volume $=15 \&$ issue $=3 \&$ spage $=259>$. Acesso em: 10 jan. 2017.

KURTZ, C.; ERNANI, P. R. Produtividade de cebola influenciada pela aplicação de micronutrientes. Revista Brasileira de Ciência do Solo, v. 34, n. 1, p. 133-142, fev. $2010 . \quad$ Disponível em: $<$ http://www.scielo.br/scielo.php?script=sci_arttext\& pid $=$ S0100 -

$06832010000100014 \& \operatorname{lng}=$ en\&nrm $=$ iso\&tlng $=\mathrm{pt}>$.

Acesso em: 16 set. 2014.
KURTZ, C.; ERNANI, P. R. Produtividade de cebola influenciada pela aplicação de micronutrientes. Revista Brasileira de Ciência do Solo, v. 34, n. 1, p. 133-142, fev. $2010 . \quad$ Disponível em: $<$ http://www.scielo.br/scielo.php?script=sci_arttext\& pid $=$ S0100

$06832010000100014 \& \operatorname{lng}=$ en\&nrm $=$ iso\&tlng $=$ pt $>$. Acesso em: 03 abr. 2017.

KURTZ, C.; ERNANI, P. R.; PAULETTI, V.; MENEZES JUNIOR, F. O. G.; VIEIRA NETO, J. Produtividade e conservação de cebola afetadas pela adubação nitrogenada no sistema de plantio direto. Horticultura Brasileira, v. 31, n. 4, p. 559-567, dez. 2013. Disponível $<$ http://www.scielo.br/scielo.php?script=sci_arttext\& pid $=$ S0102

05362013000400009\&lng=en\&nrm=iso\&tlng=pt $>$. Acesso em: 30 set. 2014.

KURTZ, C.; PAULETTI, V.; FAYAD, J. A.; VIEIRA NETO, J. Crescimento e absorção de nutrientes pela cultivar de cebola Bola Precoce. Horticultura Brasileira, v. 34, n. 2, p. 279-288, jun. 2016. Disponível em: $<$ http://www.scielo.br/scielo.php?script=sci_arttext\& pid $=$ S0102-

05362016000200279\&lng=en\&nrm $=$ iso\&tlng=pt $>$.

Acesso em: 31 mar. 2017.

LEE, J. Effect of application methods of organic fertilizer on growth, soil chemical properties and microbial densities in organic bulb onion production. Scientia Horticulturae, v. 124, n. 3, p. 299-305, abr. 2010. Disponível em: <http://www.sciencedirect.com/science/article/pii/S 0304423810000075 >. Acesso em: 31 mar. 2017.

LEE, J.; HWANG, S.; LEE, S.; HA, I.; HWANG, H.; LEE, S.; KIM, J. Comparison Study on Soil Physical and Chemical Properties, Plant Growth, Yield, and Nutrient Uptakes in Bulb Onion from Organic and Conventional Systems. HortScience, v. 49, n. 12, p. 1563 1567, 1 dez. 2014. Disponível em: $<$ http://hortsci.ashspublications.org/content/49/12/1 563.abstract>. Acesso em: 18 ago. 2015.

MALAVOLTA, E.; VITTI, G. .; OLIVEIRA, S. A. Avaliação do estado nutricional das plantas: princípios e aplicações. Piracicaba: Associação Brasileira para Pesquisa da Potassa e do Fosfato, 1989. 
MENEZES JÚNIOR, F. O. de; GONÇALVES, P. A. S.; KURTZ, C. Biomassa e extração de nutrientes da cebola sob adubação orgânica e biofertilizantes. Horticultura Brasileira, v. 31, n. 4, p. 642-648, dez. 2013. Disponível em: $<$ http://www.scielo.br/scielo.php?script=sci_arttext\& pid=S0102-

$05362013000400022 \& \operatorname{lng}=$ en\&nrm $=$ iso\&tlng $=\mathrm{pt}>$.

Acesso em: 30 set. 2014.

MENEZES JÚNIOR, F. O.; GONÇALVES, P. A.; VIEIRA NETO, J. Produtividade da cebola em cultivo mínimo no sistema convencional e orgânico com biofertilizantes. Horticultura Brasileira, v. 32, n. 4, p. 475481, dez. 2014. Disponível em: $<$ http://www.scielo.br/scielo.php?script=sci_arttext\& pid=S0102-

05362014000400475\&lng=pt\&nrm=iso\&tlng=en $>$.

Acesso em: 11 nov. 2014.

MENEZES JÚNIOR, F. O. de; KURTZ, C. Produtividade da cebola fertirrigada sob diferentes doses de nitrogênio e densidades populacionais. Horticultura Brasileira, v. 34, n. 4, p. 571-579, dez. 2016. Disponível em: http:/ / www.scielo.br/scielo.php?pid=S0102$05362016000400571 \&$ script $=$ sci_abstract\&tlng =pt > Acesso em: 13 mar. 2017.

NUNES, C. A.; FREITAS, M. P.; PINHEIRO, A. C. M.; BASTOS, S. C. Chemoface: a novel free userfriendly interface for chemometrics. Journal of the Brazilian Chemical Society, v. 23, p. 2003-2010, 2012. Disponível em: $<$ http://www.scielo.br/scielo.php?script=sci_arttext\& pid $=$ S0103$50532012005000073 \& \operatorname{lng}=$ en\&nrm $=$ iso\&tlng $=$ en $>$. Acesso em: 11 jan. 2013.

SILVA, C. A. Uso de resíduos orgânicos na agricultura. In: SANTOS, G. DE A.; SILVA, L. S. DA;
CANELlAS, L. P.; CAMARGO, F. A. DE O. (Ed.). Fundamentos da matéria orgânica do solo: ecossistemas tropicais \& subtropicais. 2. ed. Porto Alegre: Metropole, 2008. p. 597-624.

SOUZA, M.; COMIN, J. J.; LEGUIZAMÓN, E. S.; KURTZ, C.; BRUNETTO, G.; MÜLLER JÚNIOR, V.; VENTURA, B.; CAMARGO, A. P. Matéria seca de plantas de cobertura, produção de cebola e atributos químicos do solo em sistema plantio direto agroecológico. Ciência Rural, v. 43, n. 1, p. 21-27, jan. $2013 . \quad$ Disponível em: $<$ http://www.scielo.br/scielo.php?script=sci_arttext\& pid $=$ S0103-

84782013000100004\&lng $=$ en\&nrm $=$ iso\&tlng $=\mathrm{pt}>$. Acesso em: 30 set. 2014.

SOUZA, R. B.; ALCÂNTARA, F. A. Adubação no sistema orgânico de produção de hortaliças. Brasília: Embrapa Hortaliças. 8p, 2008. (Circular Técnica, 65). Disponível em:

<http://www.cnph.embrapa.br/paginas/serie_docume ntos/publicacoes2008/ct_65.pdf >.

VIDIGAL, S. M.; SEDIYAMA, M. A. N.; PEDROSA, M. W.; SANTOS, M. R. Produtividade de cebola em cultivo orgânico utilizando composto à base de dejetos de suínos. Horticultura Brasileira, v. 28, n. 2, p. 168-173, jun. 2010.2 Disponível em: $<$ http://www.scielo.br/scielo.php?script=sci_arttext\& pid $=$ S0102

$05362010000200005 \& \operatorname{lng}=$ en\&nrm $=$ iso\&tlng $=$ pt $>$. Acesso em: 10 jun. 2015.

WESTERMAN, P. W.; BICUDO, J. R. Management considerations for organic waste use in agriculture. Bioresource Technology, v. 96, n. 2, p. 215-221, jan. 2005. Disponível em: <http://www.ncbi.nlm.nih.gov/pubmed/15381219>. Acesso em: 26 jul. 2011. 